\title{
Disorders noticed during development of pancreatic cancer: potential opportunities for early and effective diagnostics and therapy
}

\author{
BARBARA JOANNA BAEAN ${ }^{I}$, EWA ZYGMANOWSKA ${ }^{l}$, \\ DOROTA MAGDALENA RADOMSKA-LEŚNIEWSKA ${ }^{2}$ \\ ${ }^{1}$ Department of Immunology, Biochemistry and Nutrition, Medical University of Warsaw, Poland \\ ${ }^{2}$ Department of Histology and Embryology, Biostructure Centre, Medical University of Warsaw, Poland
}

\begin{abstract}
Pancreatic cancer, with a total five-year survival rate below 5\%, represents a disease with a high level of malignancy. Some of the pancreatic cancer bad prognosis factors are nutrition disorders. Malnutrition, neither recognized nor properly referred to by the healthcare system, leads to well-documented negative health consequences in hospitalized patients including their impaired immunity, delayed post-surgery wound healing, a high risk of infectious complications, morbidity and mortality. There are numerous factors contributing to the development of pancreatic cancer, including telomerases, inflammation, angiogenesis, epigenetics and genetics factors, miRNA, pancreatic cancer stem cells. On the basis of molecular analyses, it has been established that precursor injuries may trigger pancreatic cancer when added to genetic alterations. Perhaps, combination of few presently used methods, like signal transduction modulated by K-ras, STAT3 activation, HMGB1 releasing, presence of oxidative stress and free radicals secretion, genes for proangiogenic growth factors activation or tissue-specific miRNA genes expression - will solve the problem of inadequate diagnostics.
\end{abstract}

Key words: pancreatic cancer, malnutrition, tumour angiogenesis, oxidative stress, miRNA, inflammation, high-mobility group box 1 (HMBG1).

(Centr Eur J Immunol 2017; 42 (4): 377-382)

\section{Introduction}

Pancreatic cancer, with a total five-year survival rate below $5 \%$, represents a disease with a high level of malignancy. Surgical resection constitutes the sole therapeutic strategy; however, the chances of successful tumour resection are seriously limited due to the advanced stage of disease development in patients, frequently with distant metastases. Present diagnostics is often unable to early detect the disease, whereas at more advanced stages, available therapeutic strategies are ineffective. Due to the above, a necessity to develop new diagnostic tools and treatment strategies has arisen $[1,2]$.

In case of cancer patients, especially cancer of the gastro-intestinal tract, both obesity and malnutrition, the latter more frequently, are perceived as essential issues to be tackled both in the medical and public health aspect. Malnutrition results in repeated hospital stays, slower recovery processes, increased number of complications in infections, bad respiration and tumour progression. All of them result in an elevated patient death rate. It is assessed that one deals with malnutrition in as many as 10 to 40 per cent of hospital-admitted cases and nearly 100 percent in case of patients diagnosed with other cancer varieties. The number of patients diagnosed with pancreatic cancer, often at an advanced stage, exceeds 60,000 per year in Europe alone. Potentially curable localised tumours are confirmed in less than $20 \%$ of cases. Elderly persons suffer from pancreatic cancer more often than younger ones [1-3].

Pancreatic cancer is divided into sub-types on the basis of the functions of pancreas it affects. Exocrine and endocrine function-related tumours differ in their causing factors, symptoms, treatment, prognoses and potential risk factors. The tumour that affects exocrine functions is benign (cystadenomas) or adenocarcinomas in $95 \%$ of cases. It appears in gland cells or pancreatic enzyme cells. Ampullary cancer

Correspondence: Dorota Magdalena Radomska-Leśniewska, Department of Histology and Embryology, Biostructure Centre,

Medical University of Warsaw, Warsaw, Poland, e-mail: dradomska@wum.edu.pl

Submitted: 6.06.2017; Accepted: 2.08.2017 
starts where the bile duct and pancreatic duct cross the duodenum in the ampulla of Vater. Islet cell cancers (neuroendocrine) are the tumours affecting endocrine functions of the pancreas [2-4].

Although several factors causing pancreatic cancer have been indicated - among which tobacco use remains the only scientifically proven one - the origin of pancreatic cancer is not known. Alcohol, coffee and aspirin intake are considered possible contributing factors, but establishing their detailed role in the process requires further research. Chronic cirrhosis, a diet rich in cholesterol and fat, and previous cholecystectomy seem to increase the probability of developing pancreatic cancer; diabetes or chronic pancreatitis are quoted as increasing incidence. It has been well-established that the risk of pancreatic cancer is 57 times higher in families with at least four affected members than in the ones with no affected members. The mechanism has not been explained yet, though it has been confirmed that the high-risk group have germ-line mutations of DNA repair genes such as BRCA2 and the partner and localizer of BRCA2 (PALB2). For that reason, it is crucial to discover the mechanisms causing the development and progression of pancreatic cancer especially at molecular levels in order to create methods of early detection and effective treatment of patients [3-5].

\section{Nutritional status assessment}

Some of the pancreatic cancer bad prognosis factors are nutrition disorders. Malnutrition, neither recognised nor properly referred to by the healthcare system, leads to well-documented negative health consequences in hospitalised patients including their impaired immunity, delayed post-surgery wound healing, a high risk of infectious complications, morbidity and mortality. The above prolong hospital stays and increase health care costs. It has been documented that malnutrition among hospitalized patients affects from $15 \%$ to even $60 \%$ worldwide, which constitutes a factor facilitating integration of nutritional support teams with the other health care representatives [6-8].

Malnutrition assessment, as a means allowing to identify patients facing or suffering from malnutrition, has been recommended by the American Society for Parenteral and Enteral Nutrition (ASPEN), European Society for Parenteral and Enteral Nutrition (ESPEN), Chinese Society for Parenteral and Enteral Nutrition (CSPEN) and Japanese Society for Parenteral and Enteral Nutrition (JSPEN) [6-8].

A standard for nutritional status assessment of hospitalized patients has not yet been developed, therefore a number of methods based on different indicators (biochemical tests, anthropometric indices) are in use, among them the Subjective Global Assessment of Nutritional Status (SGA), Mini Nutritional Assessment (MNA), Nutritional Risk Screening (NRS 2002-2012) [7, 8].

\section{Oncogene activation mechanisms}

There are numerous factors contributing to the development of pancreatic cancer, among them telomerases, inflammation, angiogenesis, epigenetics and genetics factors, miRNA, pancreatic cancer stem cells. On the basis of molecular analyses, it has been established that precursor injuries may trigger pancreatic cancer when added to genetic alterations [4].

The Pancreatic Intraepithelial Neoplasia (PanIN) scheme is used for classifying lesions. PanIN are microscopic lesions in the smaller pancreatic ducts. They are divided into four categories: PanIN-1A, PanIN-1B, PanIN-2 and PanIN-3. The most important gene changes with gain of function belonging to PanIN: RAS, EGFR, COX-2, HGF, FADK, Notch, Wnt, Myb, CCKRB, BCL-6, CXCR4, AKT-2, and the genes with loss of function promote pre-cancerous lesions: BRCA2, PTEN, SMAD4, p16, p53, p21/WAF, p27/ CIP1. Intraductal papillary mucinous neoplasms (IPMNs), in the form of cysts in pancreas possible to be detected radiologically, constitute another precursor [4].

Most research concentrates on identification of molecular events connected with pancreatic carcinogenesis and their relation to the pathological status. Numerous subsets of genes become either activated or inactivated in the course of pancreatic cancer development and progression. Oncogene activation and tumour-suppressing gene inactivation are partly responsible for the beginning of the disease. Molecular deregulation in a number of signalling pathways is of importance in this case [9, 10].

Different mechanisms activate oncogenes. One of them is based on point mutation and amplification. RAS oncogene activation is observed in more than $90 \%$ of pancreatic cancer cases. RAS genes encode three membrane-bound proteins participating in signal transduction and mediating pleiotropic effects, among them cell proliferation and migration. Activated RAS is engaged in growth factor-mediated signal transduction pathways. It is estimated that from $80 \%$ to $90 \%$ of pancreatic cancer cases are rooted at point mutations at codons 12, 13, and 61 in K-ras. Activated RAS joins GTP and sends haphazard stimulation signals to downstream signalling cascades, this way facilitating uncontrolled cell growth. K-ras mutations in pancreatic cancer appear mostly in the early phase of carcinogenesis. In such cases amplification of RAS is also observed [9-11].

\section{Inflammation}

Inflammation is currently perceived a factor leading to the development of pancreatic cancer. A number of components, among them cytokines such as TNF- $\alpha$, IL-6, IL-8, interferon- $\gamma$, the COX-2, and the peroxisome proliferator-activated receptor- $\gamma$, which function in the inflammatory response, impact initiation, promotion and progression of tumours. Other mechanisms in the inflammatory process, such 
as free radicals, may facilitate cancer development. Chronic hereditary pancreatitis, obesity, type II diabetes and smoking constitute risk factors enhancing these pathologies [12-15].

A mutated Kras-dependent mouse model, in which the animals develop PanIN lesions spontaneously, confirms the influence of inflammation in developing pancreatic cancer from PanIN lesions. Cyclical caerulein-induced acute pancreatitis may cause cell transformation by blocking the mutated K-ras-induced senescence. STAT3 activation in pancreas cancer is connected with tumour development by means of the mechanisms affecting tumour and modulating tumour-associated stroma and the immune system. Activation is determined by phosphorylation of a conserved tyrosine residue (Y705) by upstream kinases, e.g. Janus kinase 2 (JAK2). JAK2 activation demands activation of the ubiquitously expressed gp130 receptor by specific ligands (IL-6, LIF, IL-11, oncostatin M, CNTF, and IL-27). The activity of STAT3 is crucial in K-ras-induced pancreatic tumour genesis supporting the connection between STAT3 activation and cell transformation $[13,14]$.

\section{Oxidative stress}

Oxidative stress, an imbalance between production of reactive oxygen species (ROS) and antioxidant mechanisms, is a characteristic feature of many cancers [15]. Generally, cancer cells display a high level of ROS which intensify the tumour metabolic adaptation, proliferation, survival, and angiogenesis. Pancreatic cancer cells also produce a high level of ROS. In normal conditions, an excessive level of ROS is harmful to cells and the organism. However, an increased level of ROS in tumour microenvironment implicates adaptation of antioxidants capacity, meaning that a low to moderate level of ROS may enhance the proliferation of cancer cells by acting as signalling molecules or promoting the mutation of genomic DNA [16]. Elevated ROS are able to elicit genetic alterations and post-translational modifications of key cancer-related proteins. ROS and reactive nitrogen intermediates can lead to oxidative damage and nitration of DNA bases, this way increasing the risk of DNA mutations. ROS production can be induced by oncogenes as over $90 \%$ of pancreatic cancer patients exhibit K-Ras mutation [17]. It is known that NOX 4 is responsible for an increase in ROS production and cancer progression [18]. ROS-dependent progression of pancreatic cancer involves promoting of cell survival and proliferation [17], inducing endothelial cells proliferation via IL-8 increase [19], triggering invasion and metastasis by EMT transition as well as elevated expression of metalloproteinases [18].

\section{Angiogenesis}

Angiogenesis is necessary for solid tumour growth. Tumour angiogenesis is induced probably mainly by hydrogen peroxide $\left(\mathrm{H}_{2} \mathrm{O}_{2}\right)$ produced by cancer cells themselves
[20]. Activation of NOX 1 and/or de novo synthesis via defective respiration by-product of oxidative metabolism seems to be the main mechanism of ROS (mainly $\mathrm{H}_{2} \mathrm{O}_{2}$ ) generation in the tumour [21].

There is a close relationship between the ROS and angiogenesis. An increased level of ROS during hypoxia is an important factor for angiogenesis induction. Hypoxia stimulates NOX-1 and enables further ROS production triggering HIF-1 and switch on the proangiogenic genes such as VEGF [20]. Angiogenesis is mostly mediated by the VEGF family of proteins and receptors. Hypoxia and some growth factors (e.g. TGF- $\beta$, EGF), oncogenic proteins such as Ras, constitute the stimuli upregulating VEGF expression. VEGF is overexpressed in more than $90 \%$ of pancreatic cancer cases [22].

\section{miRNA expression}

Tissue-specificity of miRNA expression and its deregulation in pancreatic cancer is well-established. Currently biosynthesis, function, and the molecular regulation of miRNAs in normal physiological and pathological conditions in chronic diseases - among them various cancer sub-types - are in focus. It seems that miRNA seriously influences cell growth, differentiation and apoptosis via regulating specific gene expression [23-26].

The miRNAs are a naturally occurring, non-coding small RNAs highly specific for developmental stages [23-26]. Abnormal miRNAs expression has been identified in different cancer types, including pancreatic cancer. In case of pancreatic cancer, some miRNAs play a role of oncogenic mediators inhibiting the expression of tumour suppressors, which leads to the development and progression of pancreatic cancer. Some miRNAs though act as tumour suppressors down-regulating oncogene expression, this way suppressing cancer growth and aggressiveness. Pancreatic precancerous and cancerous cells had substantially raised oncogenic miRNAs and lowered tumour-suppressive miRNAs levels, which indicates that abnormal miRNAs expressions in association with cellular signal transduction disorder form the molecular basis of pancreatic cancer development and progression [27, 28].

Every single miRNA has hundreds of different conserved or non-conserved targets, therefore modifications in the level of a specific miRNA may result in significant changes in the expression of many genes, this way facilitating atypical changes in numerous cellular signal transduction pathways. The above leads to the development and progression of pancreatic cancer. For that reason, focusing on miRNAs might constitute an effective method of pancreatic cancer prevention/treatment by means of multiple gene regulation $[17,27,28]$.

Observing differential miRNA expression could constitute a functional tool in diagnosing pancreatic cancer. Expression of the miR-376 precursor was highest in the 
human pancreatic cancer cell line Panc1 in comparison with other cell lines studied [28, 29]. miRNA profiling with the use of 540 samples of solid tumours (stomach, lung, breast, colon, prostate and pancreas) exhibited that the spectrum of miRNA expression was different in different solid tumours and varied from normal cells. In case of colon, stomach, prostate, and pancreatic cancers, miR-218-2 was consistently downregulated, in contrast with lung and breast carcinomas [29, 30]. The above shows that colon, pancreas, prostate and stomach cancer have similar miRNA signatures. Analogically, up-regulation of miR-142-3p, miR-142-5p, miR-155 and miR-146a expressions was confirmed in human pancreatic neuroendocrine tumours (PNETs) in contrast to normal human islets [30, 31]. Lee et al. [32] noted abnormal expression of 100 miRNA precursors in pancreatic cancer as well as in miRNAs earlier reported in other types of human cancers (miR-155, miR-21, miR-221, and miR-222), just like the first reported miR-376a and miR-301 for the differential expression of cancer. Considerable upregulation of miR196a, miR-190, miR-186, miR-221, miR-222, miR-200b, miR-15b and miR-95 was documented in case of the majority of pancreatic cancer tissues and cell lines [32, 33]. miR-155 and miR-21 were upregulated considerably in 15 IPMNs versus matched controls. The expression of miR-216 has proven to be pancreas-specific [34-36].

The expression levels of miR-16, miR-21, miR-155, miR-181a, miR-181b, miR-196a and miR-210 were noticeably raised in plasma from pancreatic cancer patients in contrast to pancreatitis patients and normal adults. What is more, the raised levels of miR-16 and miR-196a as well as the raised CA19-9 serum level are more efficient in differentiating between pancreatic cancer, pancreatitis and normal individuals. The study shows as well that combined biomarkers were extremely effective in diagnosing pancreatic cancer at stage 1 , which indicates their importance for early detection of pancreatic cancer in tumour screening [23, 37]. Schultz et al. [37] showed that expression levels of 38 miRNAs were considerably changed in case of pancreatic cancer patients. On the basis of the above, two diagnostic concepts were developed: the first one included miR-145, miR-150, miR-223, and miR-636; whereas the other one was composed of miR-26b, miR-34a, miR-122, miR-126, miR-145, miR-150, miR-223, miR-505, miR-636 and miR-885. The sensitivities and specificities in case of these two sets were lower than in case of CA19-9. When they were connected with CA19-9, their sensitivity and specificity when it comes to diagnosing pancreatic cancer was highly improved and showed that circulating miRNA detection in connection with CA19-9 measurement could help to identify pancreatic cancer early [23, 34-38]. A study was conducted to identify serum miRNAs that could be used to identify patients with low-stage pancreatic cancer among healthy controls and proved that serum miR-1290 was most successfully used from among all the miRNAs tested. The discrimination by miR-1290 was more effective than in case of CA19-9, which implies that testing for plasma miR-1290 might facilitate early detection of pancreatic cancer [38].

miRNAs play important roles in various types and development stages of pancreatic tumours and in many diseases, including cancers and immune disorders. Epigenetic modifications, DNA copy number changes and genetic mutations can regulate miRNAs expression. PDAC miRNA comes connected with normal and tumour tissues vary. These differences are tumour-specific and in certain cases might serve as indicators of prognosis. This suggests that miRNA expression patterns form a kind of a signature of the disease, which might trigger new theories regarding pancreatic cancer occurrence and provide new molecular markers to improve diagnosis and treatment. Promising diagnostic methods could be designed with the use of miRNAs if they are identified in serum and their presence can be confirmed by RT-PCR. Lu et al. [39] indicated that expression data for 217 miRNAs had better results in identifying cancer types than analysis of 16000 mRNAs. They concluded that miRNAs might help identify cancer better than other currently available methods as there are only several hundreds of miRNAs, whereas there are tens of thousands of mRNAs and proteins [39, 40].

\section{HMGB1}

Although the fact of releasing cell death biomarkers in the course of cancer diseases is widely quoted in the literature, its importance for immunology and patient treatment requires further research. Chemotherapy is confirmed to stimulate the immunological system of the patient as a side effect of the treatment. Continuous stimulation of the tumour surroundings however causes immune paralysis enhancing tumour growth and invasiveness. Producing damage-associated molecular pattern (DAMP) molecules, e.g. adenosine triphosphate or high-mobility group box 1 (HMGB1) joining diverse surface receptors on immune cells leads to inflammation [41-44].

HMGB1 is a nuclear protein playing a role of an architectural chromatin-binding agent participating in constructing and preserving nucleosome structure and regulating gene transcription. It is built of 215 amino acids arranged in two spherical DNA-binding domains, box A and box B, and with an acidic C-terminal tail. It is produced either by immune cells (active secretion) or in case of cellular damage, e.g. cell death (passive secretion). Different factors stimulate its release into extracellular milieu, where it facilitates development of manifold prolonged inflammatory and autoimmune diseases, among them cancer and sepsis [43-45]. One of the symptoms of the development of these diseases is interaction of the freed HMGB1 with the receptor for advanced glycation end products (RAGE) located on the surface of the cell $[44,46]$. Moreover, these are members of the Toll-like family of receptors (TLRs), among 
them TLR2, TLR4 and TLR9. Their stimulation leads to activation of $\mathrm{NF}-\kappa \mathrm{B}$, which in turn impacts the release of cytokines, this way facilitating inflammation. $\mathrm{NF}-\kappa \mathrm{B}$, activation triggers HMGB1 receptors and increases secretion of HMGB1 by an optimistic reaction of loop. HMGB1 participates in numerous biologically vital processes, e.g. transcription, recombination, DNA repair and extracellular signalling. HMGB is composed of two other nuclear factors, HMGB2 and HMGB3. HMGB1 is commonly expressed in all vertebrate nuclei, however the expressions of HMGB2 and HMGB3 are more coordinated. HMGB1 is secreted by mature dendritic cells, macrophages and natural killer cells which are animated by LPS, IL- 1 or TNF- $\alpha$. It is considered an inflammatory cytokine acting as a late mediator of lethality in sepsis. HMGB1 is implied to play a role of a regulating agent in case of inflammation after acute tissue damage, burn, infection, responses of ischemia or sepsis and innate tissue regeneration [47-49].

HMGB1 is vital for effective anticancer immune responses though it may facilitate tumour growth, angiogenesis and metastasis. HMGB1 in angiogenesis acts as a pro-angiogenesis factor generating vascular endothelial growth factor (VEGF) in colon cancer, whereas RAGE was confirmed to be necessary for cell angiogenesis in hepatocellular carcinoma. Targeted suppression of RAGE or HMGB1 caused intensified apoptosis and lowered viability of pancreatic tumour cells increasing at the same time their sensitivity to anticancer chemotherapy. The above results from inhibiting HMGB1/RAGE-dependent pathway sustaining autophagy as a key tumour survival mechanism [50-56].

\section{Conclusions}

Pancreatic cancer is one of the worst cancers all over the world. Unfortunately, we still do not have one effective method of early diagnosis of the disease. We believe that a combination of few presently used methods, like signal transduction modulated by K-ras, STAT3 activation, HMGB1 releasing, markers of oxidative stress and free radicals secretion, genes for proangiogenic growth factors activation or tissue-specific miRNA genes expression will solve the problem of inadequate diagnostics.

The authors declare no conflict of interest.

\section{References}

1. Hidalgo M (2010): Pancreatic cancer. N Eng J Med 362: 1605-1617.

2. Chang JC, Kundranda M (2017): Novell diagnostic and predictive biomarkers in pancreatic adenocarcinoma. Int $\mathrm{J}$ Mol Sci 18: 667-681.

3. Abreu FB, Liu X, Tsongalis GJ (2017): miRNA analysis in pancreatic cancer: the Dartmouth experience. Clin Chem Lab Med 55: 755-762.
4. Lovanna J, Mallmann MC, Goncalves A, et al. (2012): Current knowledge on pancreatic cancer. Frontiers in Oncology 2: $1-24$.

5. Corbo V, Tortora G, Scarpa A (2012): Molecular pathology of pancreatic cancer: from bench to beside translation. Current Drug Targets 13: 744-752.

6. Słotwiński R, Olszewski WL, Krasnodębski IW, et al. (2006): Changes in interleukin- 6 and cytokines antagonists serum concentrations in patients after pancreatic cancer surgery receiving nutritional support. Centr Eur J Immunol 31: 25-30.

7. Kondrup J, Allison SP, Elia M, et al. (2003): Educational, Clinical Practice Committee ESoP, enteral N. ESPEN guidelines for nutrition screening 2002. Clin Nutr 22: 415-421.

8. McClave SA, Martindale RG, Vanek VW, et al. (2009): Guidelines for the provision and assessment of nutrition support therapy in the adult critically Ill patient: Society of Critical Care Medicine and American Society for Parenteral and Enteral Nutrition. J Parenter Enteral Nutr 33: 277-316.

9. Guerran C, Schuhmacher AJ, Canamero M, et al. (2007): Chronic pancreatitis is essential for induction of pancreatic ductal adenocarcinoma by K-Ras oncogenes in adult mice. Cancer Cell 11: 291-302.

10. Singh M, Maitra A (2007). Precursor lesions of pancreatic cancer: molecular pathology and clinical implications. Pancreatology 7: 9-19.

11. Mimeault M, Brand RE, Sasson AA, et al. (2005). Recent advances on the molecular mechanisms involved in pancreatic cancer progression and therapies. Pancreas 31: 301-316.

12. Mohammed A, Janakiram NB, Pant S, et al. (2015): Molecular targeted intervention for pancreatic cancer. Cancers 7: 1499-1542.

13. Holmer R, Goumas FA, Waetzig GH, et al. (2014): Interleukin-6: a villain in the drama of pancreatic cancer development and progression. Hepatobiliary Pancreat Dis Int 13: 371-380.

14. Philip B, Roland CL, Daniluk J, et al. (2013): A high-fat diet activates oncogenic Kras and COX2 to induce development of pancreatic ductal adenocarcinoma in mice. Gastroenterology 145: $1449-1458$.

15. Zhang L, Li J, Zong L, et al. (2016): Reactive oxygen species and targeted therapy for pancreatic cancer. Oxid Med Cell Longev 2016: 1-9.

16. Almoguera C, Shibata D, Forrester K, et al. (1988): Most human carcinomas of the exocrine pancreas contain mutant c-K-ras genes. Cell 53: 549-554.

17. Ogrunc M, Di Micco R, Liontos M, et al. (2014): Oncogene induced reactive oxygen species fuel hyperproliferation and DNA damage response activation. Cell Death Differ 21: 9981012.

18. Edderkaoui M, Hong P, Vaquero EC, et al. (2005): Extracellular matrix stimulates reactive oxygen species production and increases pancreatic cancer cell survival through 5-lipoxygenase and NADPH oxidase. Am J Physiol Gastrointest Liver Physiol 289: G1137-G1147.

19. Sawai H, Funahashi H, Okada Y, et al. (2005): Interleukin $1 \alpha$ enhances IL-8 secretion through p38 mitogen-activated protein kinase and reactive oxygen species signaling in human pancreatic cancer cells. Medical Science Monitor 11: BR343BR350.

20. Radomska-Leśniewska DM, Hevelke A, Skopiński P, et al. (2016): Reactive oxygen species and synthetic antioxidants as angiogenesis modulators: clinical implications. Pharmacol Rep 68: 462-471. 
21. Kim YM, Kim KE, Koch GY, et al. (2006): Hydrogen peroxide produced by angiopoietin-1 mediates angiogenesis. Cancer Res 66: 6167-6174.

22. Kardech S, Ashkani-Esfahani S, Aliozadeh AM (2014): Paradoxical action of reactive oxygen species in creation and therapy of cancer. Eur J Pharmacol 2735: 150-168.

23. Yiwei L, Sarkar FH (2016): MicroRNA targeted therapeutic approach for pancreatic cancer. Int J Biol Sci 12: 326-337.

24. Friedman RC, Farh KK, Burge CB, et al. (2009): Most mammalian mRNAs are conserved targets of microRNAs. Genome Res 19: 92-105.

25. Lewis BP, Burge CB, Bartel DP (2005): Conserved seed pairing, often flanked by adenosines, indicates that thousands of human genes are microRNA targets. Cell 120: 15-20.

26. Winter J, Jung S, Keller S, et al. (2009): Many roads to maturity: microRNA biogenesis pathways and their regulation. Nat Cell Biol 11: 228-234.

27. Cutts RJ, Gadaleta E, Hahn SA, et al. (2011): The Pancreatic expression database: 2011 update. Nucleic Acids Res 39: D1023-D1028.

28. Munding JB, Adai AT, Maghnouj A, et al. (2012): Global microRNA expression profiling of microdissected tissues identifies miR-135b as a novel biomarker for pancreatic ductal adenocarcinoma. Int J Cancer 131: E86-E95.

29. Jiang J, Lee EJ, Gusev Y, et al. (2005): Real-time expression profiling of microRNA precursors in human cancer cell lines. Nucleic Acids Res 33: 5394-5403.

30. Volinia S, Calin GA, Liu CG, et al. (2006): A microRNA expression signature of human solid tumors defines cancer gene targets. Proc Natl Acad Sci U S A 103: 2257-2261.

31. Olson P, Lu J, Zhang H, et al. (2009): MicroRNA dynamics in the stages of tumorigenesis correlate with hallmark capabilities of cancer. Genes Dev 23: 2152-2165.

32. Lee CH, Subramanian S, Beck AH, et al. (2009): MicroRNA profiling of BRCA1/2 mutation-carrying and non-mutation-carrying high-grade serous carcinomas of ovary. PLoS One 4: e7314.

33. Siuwah Tang BS, Jillian Bonaroti BS, Sebnem U, et al. (2013): Sweating the small stuff microRNAs and genetic changes define pancreatic cancer. Pancreas 42: 740-759.

34. Feldmann G, Habbe N, Dhara S, et al. (2008): Hedgehog inhibition prolongs survival in a genetically engineered mouse model of pancreatic cancer. Gut 57: 1420-1430.

35. Altaf M, Naveena JB (2015): Molecular Targeted Intervention for Pancreatic Cancer. Cancers 7: 1499-1542.

36. Sood P, Krek A, Zavolan M, et al. (2006): Cell- type-specific signatures of microRNAs on target mRNA expression. Proc Natl Acad Sci U S A 103: 2746-2751.

37. Schultz NA, Dehlendorff C, Jensen BV, et al. (2014): MicroRNA biomarkers in whole blood for detection of pancreatic cancer. JAMA 311: 392-404.

38. Li A, Yu J, Kim H, et al. (2013): MicroRNA array analysis finds elevated serum miR-1290 accurately distinguishes patients with low-stage pancreatic cancer from healthy and disease controls. Clin Cancer Res 19: 3600-3610.

39. Lu J, Getz G, Miska EA (2005): MicroRNA expression profiles classify human cancers. Nature 435: 834-838.

40. Binker BG, Binker-Cosen AA, Richards D, et al. (2009): EGF promotes invasion by PANC-1 cells through Rac1/ROS-dependent secretion and activation of MMP-2. Biochem Biophys Res Commun 379: 445-450.
41. Kwon JJ, Nabinger SC, Vega Z, et al. (2015): Pathophysiological role of microRNA-29 in pancreatic cancer stroma. Scientific Reports 5: 11450-11472.

42. Xuanbin W, Longchao X, Hongliang L, et al. (2015): The role of HMGB1 signalling pathway in the development and progression of hepatocellular carcinoma: A review. Int J Mol Sci 16: 22527-22540.

43. Noguiera-Machado JA, de Oliviera Volpe CM (2012): HMGB-1 as a target for inflammation controlling. Recent Pat Endocr Metab Immune Drug Discov 6: 201-209.

44. Rojas A, Delgado-Lopez F, Perez-Castro R, et al. (2016): HMGB-1 enhances the protumoral activities of M2 macrophages by a RAGE-dependent mechanism. Tumour Biol 37: 3321-3329.

45. Xu YL, Qian QL, Zong LZ, et al. (2014): High mobility group Box1 (HMGB1) is associated with progression and poor prognosis in pancreatic cancer. Gastroint Dig Syst 4: 1-7.

46. Nativel B, Marimouton M, Thon-Hon V, et al. (2013): Soluble HMGB1 is a novel adipokine stimulating IL-6 secretion through RAGE receptor in SW872 preadipocyte cell line: Contribution to chronic inflammation in fat tissue. PLoS One 8: $1-11$.

47. Kang R, Zhang Q, Hou W, et al. (2014): Intracellular HMGB1 inhibits inflammatory nucleosome release and limits acute pancreatitis in mice. Gastroenterology 146: 1097-1107.

48. Musumeci D, Roviello GN, Montesarchio D (2014): An overview on HMGB-1 inhibitors as potential therapeutic agents in HMGB-1-related pathologies. Pharmacol Ther 141: 347-357.

49. Kang R, Tang D, Schapiro NE, et al. (2014): The HMGB1/ RAGE inflammatory pathway promotes pancreatic tumor growth by regulating mitochondrial bioenergetics. Oncogene 33: 567-577.

50. Angelopoulou E, Piperi C, Adamopoulos C, et al. (2016): Pivotal role of high-mobility group box 1 (HMGB1) signalling pathways in glioma development and progression. $\mathrm{J}$ Mol Med (Berl) 94: 867-874.

51. Sharma S, Evans A, Hemers E (2016): Mesenchymal-epithelial signalling in tumour microenvironment: role of high-mobility group Box-1. Cell Tissue Res 365: 357-366.

52. Van Beijnum JR, Buurman WA, Griffioen AW (2008): Convergence and amplification of toll-like receptor (TLR) and receptor for advanced glycation end products (RAGE) signalling pathways via high mobility group B1 (HMGB1). Angiogenesis 11: 91-99.

53. Kishikawa T, Otsuka M, Ohno Motoko, et al. (2015): Circulating RNAs as new biomarkers for detecting pancreatic cancer. World J Gastroenterol 21: 8527-8540.

54. Shing-Chun T, Yang-Chao Ch (2014): Novel therapeutic targets for pancreatic cancer. World J Gastroenterol 20: 1082510844.

55. Inman KS, Francis AA, Murray NR (2014): Complex role for the immune system in initiation and progression of pancreatic cancer. World J Gastroenterol 20: 11160-11181.

56. Wittwer Ch, Boeck S, Heinemann V, et al. (2013): Circulating nucleosomes and immunogenic cell death markers HMGB1, sRAGE and DNAse in patients with advanced pancreatic cancer undergoing chemotherapy. Int J Cancer 133: 2619-2630. 